\title{
AFFINELY REGULAR POLYGONS AS EXTREMALS OF AREA FUNCTIONALS
}

\author{
PAOLO GRONCHI AND MARCO LONGINETTI
}

\begin{abstract}
For any convex $n$-gon $P$ we consider the polygons obtained dropping a vertex or an edge of $P$. The area distance of $P$ to such $(n-1)$-gons, divided by the area of $P$, is an affinely invariant functional on $n$-gons whose maximizers coincide with the affinely regular polygons. We provide a complete proof of this result.

We extend these area functionals to planar convex bodies and we present connections with the affine isoperimetric inequality and parallel X-ray tomography.
\end{abstract}

\section{INTRODUCTION}

Given a convex polygon $P$ with $n$ vertices $z_{j}$ ordered counterclockwise, we define $W_{j}(P)$ to be the triangle $z_{j-1} z_{j} z_{j+1}$ and $T_{j}(P)$ to be the (possibly infinite) triangle outside $P$ bounded by the side $z_{j} z_{j+1}$ and the continuations of its two adjacent sides (see Fig. 1). Henceforth, the index $j$ is taken modulo $n$, and $|C|$ denotes the area of $C$.

In this paper we consider the following affinely invariant functionals defined on the class $\mathcal{P}_{n}$ of planar convex $n$-gons, i.e., polygons with exactly $n$ vertices:

$$
F(P)=\min _{j=1, \ldots, n} \frac{\left|W_{j}(P)\right|}{|P|}
$$

$$
G(P)=\min _{j=1, \ldots, n} \frac{\left|T_{j}(P)\right|}{|P|},
$$

and we are interested in the maximizers of these functionals.

In Theorem 1.8 it is shown that the maximizers of the above functionals are affinely regular $n$-gons, i.e., affine images of regular $n$-gons. This class, denoted by $\mathcal{R}_{n}$, often appears in geometric problems with affine invariance [1], [18], [3], [4], [16].

A characterization of $\mathcal{R}_{n}$ as extremals of area functionals was obtained by Renyi and Sulanke [18]. They proved in Satz 2 that $\prod_{i=1}^{n}\left|W_{i}(P)\right| /|P|^{n}$ attains its maximum on $\mathcal{R}_{n}$.

The functional $F$ was first introduced by Lopez and Reisner [16] in connection with algorithms for the approximation of a convex set by polygons. They showed that Theorem 1.8 for the functional $F$ is a consequence of the result by Renyi and Sulanke.

The functional $G$ was first introduced by Longinetti [14], where Theorem 1.8 is proved for $n=5,6$, via elementary geometric arguments. The functional $G$ and a similar functional

1991 Mathematics Subject Classification. Primary: 52A40.

Key words and phrases. affinely regular polygons, geometric tomography, affine lenght. 
(not affinely invariant) considered in [13] are related to Hammer's X-ray problem for planar convex bodies proposed in [9]: How many X-ray pictures of a convex body must be taken in order to permit its reconstruction? The solution of this problem is given by Gardner and McMullen [5]. We refer to [6, Ch. 1] for an overview of this topic. In Section 6 we present in detail the connection between the functional $G$ and the stability of the reconstruction in the Hammer's problem. In Section 5, we discuss some extensions of functionals $F$ and $G$ to the class of planar convex bodies related to the affine length of a convex body and to the affine isoperimetric inequality. These functionals are also related to the approximation of planar convex bodies by polygons ([8], [16]). In particular, $F$ is related to the approximation of an $n$-gon $P$ by $(n-1)$-gons contained in $P$. Similarly, $G$ is related with the approximation of $P$ by $(n-1)$-gons containing $P$. Because of this we use the word inner or outer in connection with $F$ or $G$, respectively.

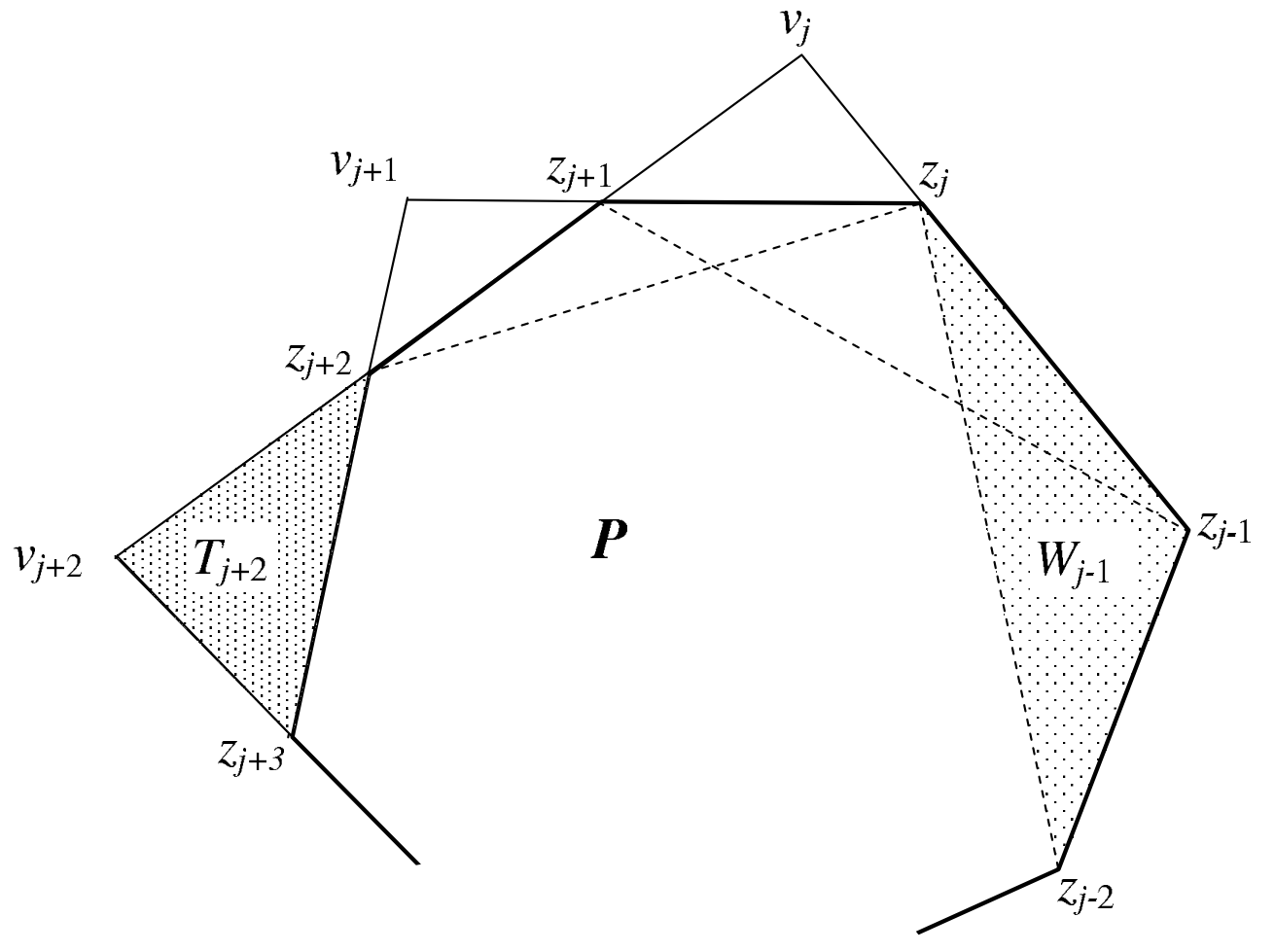

Figure 1. Triangles $W_{j}$ and $T_{j}$ of $P$.

As a first remark we deal with the trivial cases $n=3,4$. For $n=3$, we have $F(P)=1$ and $G(P)=\infty$, for every $P$. For $n=4$, by elementary arguments, one can prove that the maximizers of $F$ have diagonals which divides them into triangles of equal area, and $G(P)=\infty$ 
only for parallelograms. Hence, all maximizers of $F$ and $G$ are parallelograms, and viceversa. As mentioned, some instances of Theorem 1.8 were already proved. In this paper we complete this result in a unique frame to all $n$, for both the inner and outer functional.

We now provide a guide to the proof of Theorem 1.8. The functionals $F$ and $G$ are continuous with respect to the Hausdorff metric in $\mathcal{P}_{n}$, which is not compact, since $n$-gons can converge to polygons with fewer vertices. Hence, we have first to prove the existence of the maximizers in Lemma 2.1.

A first step towards the characterization of these maximizers is to show that they satisfy an inner (or outer) equal-area property which has some interest by itself. Consider the following classes:

$$
\begin{gathered}
\Phi_{n}=\left\{P \in \mathcal{P}_{n}:\left|W_{1}(P)\right|=\left|W_{2}(P)\right|=\cdots=\left|W_{n}(P)\right|\right\}, \\
\Gamma_{n}=\left\{P \in \mathcal{P}_{n}:\left|T_{1}(P)\right|=\left|T_{2}(P)\right|=\cdots=\left|T_{n}(P)\right|\right\} .
\end{gathered}
$$

Henceforth, we say that a polygon $P$ has the inner (outer) equal-area property when $P$ belongs to $\Phi_{n}\left(\Gamma_{n}\right.$, respectively). Section 2.1 contains the proof of the following proposition.

Proposition 1.1. F and $G$ attain their maximum on $\Phi_{n}$ and $\Gamma_{n}$, respectively.

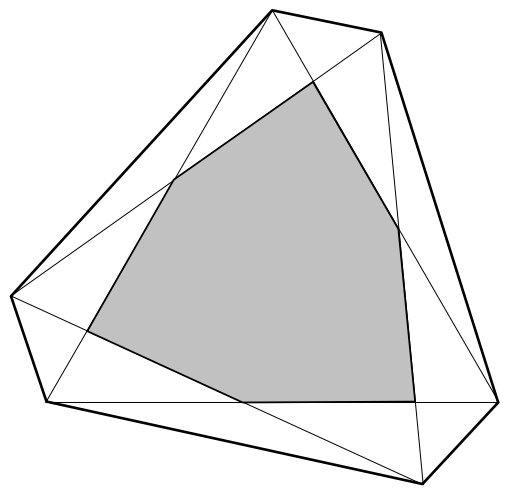

Figure 2. Polygons from $\Phi_{6}$ and $\Gamma_{6}$.

It can be proved that the classes $\Phi_{5}$ and $\Gamma_{5}$ coincide with the one of affinely regular pentagons. For $n>5$, it is easy to see that the above class is larger than the class $\mathcal{R}_{n}$ of affinely regular polygons. For example, hexagons in $\Gamma_{6}$ are, up to an affine transformation, the intersection of two concentric equilateral triangles (see the shaded polygon in Fig. 2). A proof can be found in [14]. Similarly, hexagons in $\Phi_{6}$ are, up to an affine transformation, equiangular (see the larger polygon in Fig. 2). In [10] the polygons of $\Phi_{n}$ are considered and a wider class of $\Phi_{n}$ containing not necessarily convex $n$-gons is explicitly parametrized by $n-5$ real parameters modulo the action of the affine group.

Roughly speaking, (3) or (4) are $n-1$ independent constraints. Since $n$-gons depend on the $2 n$ coordinates of its vertices, it follows that $\Phi_{n}$ and $\Gamma_{n}$ depend on $n+1$ parameters, and therefore, modulo the action of the affine group, depend on $n-5$ parameters. So, in order to 
show that all maximizers of $F$ and $G$ are in $\mathcal{R}_{n}$ other significant properties must be proved. A subclass of $\Phi_{2 m}$ was characterized by Bianchi and Longinetti in [2, Lemma 1].

Let $l_{j}$ be the length of the side $z_{j} z_{j+1}$ of $P$, i.e. $l_{j}=\left\|z_{j}-z_{j+1}\right\|$ and $d_{j}$ the length of the diagonal $z_{j-1} z_{j+2}$.

Definition 1.2. We define $\mathcal{F}_{n}$ as the class of convex n-gons such that

$$
\left(\frac{d_{j+1}-l_{j+1}}{d_{j+1}}\right)\left(\frac{d_{j}-l_{j}}{l_{j}}\right)=\left(\frac{d_{j-2}-l_{j-2}}{d_{j-2}}\right)\left(\frac{d_{j-1}-l_{j-1}}{l_{j-1}}\right) \quad \text { for } j=1, \ldots, n \text {. }
$$

We say that $P$ has the inner-ratio property when $P \in \mathcal{F}_{n}$. Assuming that all triangles $T_{j}$ of $P$ are bounded, we define $v_{j}$ as the vertex of $T_{j}$ not in $P$. Also, set $s_{j}=\left\|v_{j}-z_{j}\right\|$ and $p_{j}=\left\|z_{j+1}-v_{j}\right\|$. Let $e_{j}$ be the length of the segment joining the outer points $v_{j+1}, v_{j-1}$. Notice that $e_{j}=p_{j-1}+l_{j}+s_{j+1}$.

Definition 1.3. We define $\mathcal{G}_{n}$ as the class of convex n-gons such that

$$
\frac{s_{j} e_{j-1}}{l_{j-1}\left(p_{j-2}+l_{j-1}\right)}=\frac{p_{j} e_{j+1}}{l_{j+1}\left(s_{j+2}+l_{j+1}\right)} \quad \text { for } j=1, \ldots, n \text {. }
$$

We call this the outer-ratio property. Notice that the outer-ratio property is an equality between ratio of length of segments on the two lines through $v_{j}$ in Fig. 1.

The following theorems, proved in Sections 2.1, 2.2, are important steps toward the goal.

Theorem 1.4. If $P^{\star}$ is a maximizer of $F$ in $\mathcal{P}_{n}$, then $P^{\star} \in \mathcal{F}_{n}$.

Theorem 1.5. If $P^{\star}$ is a maximizer of $G$ in $\mathcal{P}_{n}$, then $P^{\star} \in \mathcal{G}_{n}$.

In Section 3, through an algebraic manipulation, we will prove that in (5) the ratios $\lambda_{j}=$ $d_{j} / l_{j}$ are independent of $j$. Analogously, for the outer problem, we will prove that in (6) the ratios $\zeta_{j}=s_{j} / l_{j-1}=p_{j-1} / l_{j}$ are independent of $j$. In Section 4 we go back to planar geometry and prove the following theorems.

\section{Theorem 1.6.}

$$
\Phi_{n} \cap \mathcal{F}_{n}=\mathcal{R}_{n}
$$

\section{Theorem 1.7.}

$$
\Gamma_{n} \cap \mathcal{G}_{n}=\mathcal{R}_{n} .
$$

These results permit us to obtain the goal of the paper.

Theorem 1.8. All maximizers of $F$ or $G$ on $\mathcal{P}_{n}$ are affinely regular polygons.

\section{First VARiations at EXtremal POLYGONS}

Observe that for each $P \in \mathcal{P}_{n}$ the triangles $W_{j}(P)$ have positive area, hence $F(P)>0$, but if $P$ is close to a polygon with $n-1$ vertices, then $F(P)$ is close to zero. Therefore $\inf _{P \in \mathcal{P}_{n}} F(P)=0$ and $F$ has no minimum in $\mathcal{P}_{n}$. Similarly, $\inf _{P \in \mathcal{P}_{n}} G(P)=0$ and $G$ has no minimum in $\mathcal{P}_{n}$. Then all the extremals of the functionals $F$ and $G$ in $\mathcal{P}_{n}$ are maximizers. In this section we obtain the more significant properties of these polygons. We use only 
elementary arguments of Euclidean geometry in the proof of Proposition 1.1. The idea of the proof is to consider a maximizer $P^{\star}$ of each functional and a suitable local variation $P_{\varepsilon}^{\star}$ of one or two vertices. An analysis of the sign of the area difference $\Delta_{\varepsilon}$ yields the results.

To prove directly the subsequent Theorems 2.5 and 2.7 , which are the principal goal of this section, a more complicated perturbation $P_{\varepsilon}^{\star}$ of $P^{\star}$ involving five consecutive vertices of $P^{\star}$ can be carried out. In this case one has to take into account only the first order terms of $\Delta_{\varepsilon}$. This computation, using Proposition 1.1, can be explicitly obtained in terms of the sides and angles of $P^{\star}$.

Here, we prefer to give a different proof, less geometric, via partial differentiation of area functionals (Lemma 2.2, and Lemma 2.3) with respect to the vertices $z_{j}$. This yields the Lagrange multiplier systems (21) and (27) for the area $|P|$ under the corresponding equalarea property constraints. At the end of each subsection, an algebraic manipulation of such systems will give the proof of Theorem 2.5 for the inner problem and the proof of Theorem 2.7 for the outer one.

We begin with the following result.

Lemma 2.1. The functionals $F$ and $G$ have maxima in $\mathcal{P}_{n}$.

Proof. From the well-known John theorem about the maximal ellipse contained in $P$, see [11], we can restrict to $n$-gons with fixed area 1 , and contained in a circular annulus $A$ of radii $r$ and $2 r$. Clearly $r \geq 1 / \sqrt{4 \pi}$ and the diameter of $P$ is less or equal to $D=4 / \sqrt{\pi}$. Represent each polygon in $\mathcal{P}_{n}$ as a point in $\mathbb{R}^{2 n}$ with coordinates the coordinates of its vertices. With respect to the standard metric on $\mathbb{R}^{2 n}, F$ is continuous and the class of $n$-gons with vertices contained in $A$ is compact. Since $F>0$, it is trivial to show that a convergent maximizing sequence on $\mathcal{P}_{n}$ has vertices which converge to $n$ distinct points, no three collinear.

Turning to $G$, some difficulties arise since $\left|T_{j}\right|$ may be unbounded. Let $\alpha_{j}$ be the exterior angle of $P$ at the vertex $z_{j}$. If $T_{j}(P)$ is bounded then

$$
\left|T_{j}(P)\right|=\frac{1}{2} l_{j}^{2}\left(\cot \alpha_{j-1}+\cot \alpha_{j}\right)^{-1} .
$$

The function $\cot x$ is convex in $(0, \pi / 2)$ and odd with respect to the point $\pi / 2$. Thus

$$
\cot \left(\frac{x_{1}+x_{2}}{2}\right) \leq \frac{\cot x_{1}+\cot x_{2}}{2} \quad \text { if } \quad x_{1}, x_{2}>0 \text { and } \quad x_{1}+x_{2}<\pi
$$

Since $\alpha_{j}>0$ and $\sum_{j=1}^{n} \alpha_{j}=2 \pi$, there exists $j$ such that

$$
\frac{\alpha_{j-i}+\alpha_{j}}{2} \leq \frac{2 \pi}{n}
$$

Therefore, by (7) and (8) it follows that

$$
\min _{j=1, \ldots, n}\left|T_{j}(P)\right|<\frac{1}{4} D^{2} \tan \frac{2 \pi}{n} .
$$


Hence, $G$ is bounded from above on $\mathcal{P}_{n}$, for $n \geq 5$.

Now consider a maximizing sequence $\left\{P^{m}\right\}$ of $n$-gons with vertices in the circular annulus $A$, which converges to $P^{\star}$. It remains to show that $P^{\star}$ has exactly $n$ vertices. We can suppose

$$
\min _{j=1, \ldots, n}\left|T_{j}\left(P^{m}\right)\right|>\frac{1}{2} \sup _{P \in \mathcal{P}_{n}} G(P)=\mu>0, \text { for all } m .
$$

For each $P$ with vertices in $A$, consider a triangle similar to $T_{j}(P)$ bounded by the continuations of its sides not in $P$ and a line parallel to the side $z_{j+1} z_{j}$ through the center of $A$. If $h_{j}$ denotes the altitude of $T_{j}(P)$ to the side $z_{j+1} z_{j}$, then this larger triangle has an altitude smaller than $h_{j}+2 r$ and a base larger than $2 r$. Hence

$$
\frac{l_{j}}{h_{j}} \geq \frac{2 r}{h_{j}+2 r}
$$

i.e., $l_{j} \geq\left(2 r-l_{j}\right) h_{j} / 2 r$. Since $l_{j} h_{j}=2\left|T_{j}(P)\right| \geq 2 \mu$, we deduce $l_{j}^{2} \geq\left(2 r-l_{j}\right) \mu / r$ and consequently that the sides of the polygons $P^{m}$ are uniformly larger than a positive constant. This implies that $n$ distinct points $z_{j}^{\star}$ of $P^{\star}$ are limits of the sequences of the vertices of $P^{m}$. Moreover, from (9), the limit of the area $\left|T_{j}\left(P^{m}\right)\right|$ is strictly positive. Hence, no three consecutive points $z_{j}^{\star}$ are collinear and they are all distinct vertices of $P^{\star}$.

Proof of Proposition 1.1. The claim that $G$ attains its maximum on $\Gamma_{n}$ was already proved in [14, Theorem 1]. The arguments are similar to the one we use for the functional $F$ and will not be repeated here.

For the inner case, we argue by contradiction. Assume that $P^{\star}$ is a maximizer of $F$ not in $\Phi_{n}$. Let $W_{r}$ be a triangle of maximal area among the $n$ triangles $W_{j}$. Moving the vertex $z_{r}$ towards the interior of $P^{\star}$ reduces the area of $P^{\star}$. Since $F$ cannot increase, the value min $\left|W_{j}\right|$ has to decrease. Hence, either $W_{r-1}$ or $W_{r+1}$ is of minimal area. The freedom we have in choosing the direction along which $z_{r}$ moves easily implies that they are both of minimal area. Therefore,

$$
\begin{aligned}
& \left|z_{r-1} z_{r-2} z_{r-3}\right|=\left|W_{r-2}\right| \geq\left|W_{r-1}\right|=\left|z_{r} z_{r-1} z_{r-2}\right| \\
& \left|z_{r+1} z_{r+2} z_{r+3}\right|=\left|W_{r+2}\right| \geq\left|W_{r+1}\right|=\left|z_{r} z_{r+1} z_{r+2}\right| .
\end{aligned}
$$

Then, the distance of $z_{r-3}$ from the line containing the edge $z_{r-2} z_{r-1}$ is larger or equal to that of $z_{r}$. Analogously, the distance of $z_{r+3}$ from the line containing $z_{r+2} z_{r+1}$ is larger or equal to that of $z_{r}$. From the convexity of $P^{\star}$, the triangle $U=z_{r-3} z_{r} z_{r+3}$ is ordered counterclockwise and contained in $P^{\star}$. Therefore, any movement of $z_{r}$ inside $U$ increases the area of both $W_{r-1}$ and $W_{r+1}$ and decreases the area of $P$. The maximality of $P^{\star}$ implies that $\left|W_{r}\right|$ cannot be larger than $\min \left|W_{j}\right|$.

We represent with complex variable $x_{j}+i y_{j}$ the vertices $z_{j}$, i.e. $z_{j}$ represents both a point in the plane and a complex number; so the area functionals $\left|T_{j}\right|,\left|W_{j}\right|$ and $|P|$ are real functions of complex variables $z_{j}$. We use partial derivatives of functions with respect to complex variables 
with the notation

$$
f_{\bar{z}}=\frac{\partial f}{\partial \bar{z}}=\frac{1}{2}\left(\frac{\partial f}{\partial x}+i \frac{\partial f}{\partial y}\right) .
$$

Lemma 2.2. If $T$ is the triangle with vertices $a, b, c$ ordered counterclockwise, then

$$
\frac{4}{i} \frac{\partial|T|}{\partial \bar{a}}=c-b
$$

Proof. If $a=x_{a}+i y_{a}, b=x_{b}+i y_{b}$, and $c=x_{c}+i y_{c}$ the result is obtained by an elementary computation starting from the formula

$$
\begin{gathered}
\frac{4}{i}|T|=\frac{2}{i}\left(\left(x_{c}-x_{b}\right)\left(y_{a}-y_{b}\right)-\left(x_{a}-x_{b}\right)\left(y_{c}-y_{b}\right)\right)= \\
=(c-b)(\bar{a}-\bar{b})-(\bar{c}-\bar{b})(a-b) .
\end{gathered}
$$

Lemma 2.3. Let $b, c, d$ and $e$ be the vertices of a convex quadrilateral ordered clockwise. Let abc be the triangle $T$ outside the quadrilateral bounded by bc and the continuations of the two sides eb and $d c$. The area of $|T|$ depending on $b, c, d, e$, satisfies:

$$
\begin{aligned}
-4 i|T|_{\bar{b}} & =(a-c)+(a-b)|a e| /|b e|, \\
-4 i|T|_{\bar{c}} & =(b-a)+(c-a)|a d| /|c d|, \\
-4 i|T|_{\bar{d}} & =(a-c)|a c| /|c d|, \\
-4 i|T|_{\bar{e}} & =(b-a)|a b| /|b e| .
\end{aligned}
$$

Proof. Observe that the vertex $a$ is a function of $b, c, d, e$ and each proof starts by differentiating (11) with respect to these variables. For example, in order to get (13) we have

$$
4 i|T|_{\bar{c}}=-(c-b) \bar{a}_{\bar{c}}+(\bar{c}-\bar{b}) a_{\bar{c}}+(a-b) .
$$

To compute the partial derivatives of $a$ and $\bar{a}$ with respect to $\bar{c}$ we differentiate the collinear conditions of $a$ with $b$ and $e$ and with $c$ and $d$, i.e.

$$
\begin{aligned}
(a-b)(\bar{e}-\bar{b})-(e-b)(\bar{a}-\bar{b}) & =0, \\
(a-c)(\bar{c}-\bar{d})-(c-d)(\bar{a}-\bar{c}) & =0,
\end{aligned}
$$

and we obtain

$$
\begin{aligned}
(\bar{e}-\bar{b}) a_{\bar{c}}-(e-b) \bar{a}_{\bar{c}} & =0, \\
(\bar{c}-\bar{d}) a_{\bar{c}}-(c-d) \bar{a}_{\bar{c}} & =d-a .
\end{aligned}
$$

Solving for $a_{\bar{c}}, \bar{a}_{\bar{c}}$ in the previous equations we obtain

$$
\begin{aligned}
& a_{\bar{c}}=(e-b)(d-a) /((e-b)(\bar{c}-\bar{d})-(\bar{e}-\bar{b})(c-d)), \\
& \bar{a}_{\bar{c}}=(\bar{e}-\bar{b})(d-a) /((e-b)(\bar{c}-\bar{d})-(\bar{e}-\bar{b})(c-d)) .
\end{aligned}
$$


By substituting in (16) we get

$$
\frac{4}{i} \frac{|T|}{\partial \bar{c}}=(b-a)+(d-a) \frac{(c-b)(\bar{e}-\bar{b})-(\bar{c}-\bar{b})(e-b)}{(e-b)(\bar{c}-\bar{d})-(\bar{e}-\bar{e})(c-d)} .
$$

The proof of (13) is obtained via the formula

$$
(c-b)(\bar{e}-\bar{b})-(\bar{c}-\bar{b})(e-b)=\frac{|a c|}{|c d|}((e-b)(\bar{c}-\bar{d})-(\bar{e}-\bar{b})(c-d)) .
$$

Indeed, by subtracting the left hand side of (17) from the left hand side of (19) we deduce

$$
(c-b)(\bar{e}-\bar{b})-(\bar{c}-\bar{b})(e-b)=(c-a)(\bar{e}-\bar{b}))-(\bar{c}-\bar{a})(e-b) .
$$

Since $a, c, d$ are collinear the vector $(c-a)$ is proportional to the vector $(d-c)$ by the factor $|a c| /|c d|$. This proves (19).

The proof of (12) can be obtained in a similar way or simply interchanging $b$ and $c, e$ and $d$, by a reflection. We remark that such a reflection changes the sign as in formula (11).

The proof of (14) follows from similar computations. More explicitly, by differentiating (11) with respect to $\bar{D}$ we obtain

$$
4 i|T|_{\bar{d}}=-(c-b) \bar{a}_{\bar{c}}+(\bar{c}-\bar{b}) a_{\bar{d}} .
$$

By differentiating with respect to $\bar{d}$ the constraints (17) and (18), we get

$$
\begin{aligned}
(\bar{e}-\bar{b}) a_{\bar{d}}-(e-b) \bar{a}_{\bar{d}} & =0, \\
(\bar{c}-d) a_{\bar{d}}-(c-d) \bar{a}_{\bar{d}} & =a-c .
\end{aligned}
$$

This implies

$$
\begin{aligned}
& a_{\bar{d}}=(e-b)(a-c) /((e-b)(\bar{c}-\bar{d})-(\bar{e}-\bar{b})(c-d)) \\
& \bar{a}_{\bar{d}}=(\bar{e}-\bar{b})(a-c) /((e-b)(\bar{c}-\bar{d})-(\bar{e}-\bar{b})(c-d)) .
\end{aligned}
$$

By substituting in (20) we get

$$
\frac{4}{i} \frac{|T|}{\partial \bar{d}}=(a-c) \frac{(c-b)(\bar{e}-\bar{b})-(\bar{c}-\bar{b})(e-b)}{(e-b)(\bar{c}-\bar{d})-(\bar{e}-\bar{b})(c-d)},
$$

and from (19) we prove (14). A similar argument proves (15).

\subsection{Inner case.}

Proposition 2.4. Let $P^{\star}$ be a maximizer of $F$ on $\mathcal{P}_{n}$. Then there exist real numbers $\mu_{1}, \ldots, \mu_{n}$ such that the following equations hold for the vertices of $P^{\star}$ :

$$
\left(z_{j-1}-z_{j+1}\right)=\mu_{j-1}\left(z_{j-1}-z_{j-2}\right)+\mu_{j}\left(z_{j-1}-z_{j+1}\right)+\mu_{j+1}\left(z_{j+2}-z_{j+1}\right),
$$

for $j=1, \ldots, n$. 
Proof. By Proposition 1.1, each maximizer $P^{\star}$ satisfies the inner equal-area property. Choosing a suitable affine transformation we can assume that $\left|W_{j}(P)\right|=1$ for $j=1, \ldots, n$. By the Lagrange multipliers argument we have that at $P^{\star}$ the gradient of the area functional $|P|$ is a linear combination of the gradients of the constraints $\left|W_{i}(P)\right|=1$, i.e. there exist real multipliers $\mu_{j}$ such that at $P^{\star}$,

$$
\frac{\partial|P|}{\partial \overline{z_{j}}}=\sum_{i} \mu_{i} \frac{\partial\left|W_{i}\right|}{\partial \overline{z_{j}}} \quad \text { for } j=1, \ldots, n .
$$

Since $W_{j}$ depends only on $z_{j-1}, z_{j}, z_{j+1}$ we have that

$$
\frac{\partial\left|W_{i}\right|}{\partial \overline{z_{j}}}=0 \quad \text { for } \quad i \notin\{j-1, j, j+1\} .
$$

Now we apply Lemma 2.2 to the triangles $W_{j-1}, W_{j}, W_{j+1}$ with respect to the vertex $z_{j}$ and find that

$$
\begin{aligned}
& \frac{4}{i} \frac{\partial\left|W_{j-1}\right|}{\partial \overline{z_{j}}}=z_{j-1}-z_{j-2}, \\
& \frac{4}{i} \frac{\partial\left|W_{j}\right|}{\partial \overline{z_{j}}}=z_{j-1}-z_{j+1}, \\
& \frac{4}{i} \frac{\partial\left|W_{j+1}\right|}{\partial \overline{z_{j}}}=z_{j+2}-z_{j+1} .
\end{aligned}
$$

Since $P$ can be decomposed in the disjoint subsets $W_{j}, P \backslash W_{j}$ and the latter does not depend on the vertex $z_{j}$, we have also

$$
\frac{4}{i} \frac{\partial|P|}{\partial \overline{z_{j}}}=\frac{4}{i} \frac{\partial\left|W_{j}\right|}{\partial \overline{z_{j}}}=z_{j-1}-z_{j+1} .
$$

The five previous equations prove (21).

The system (21) is a system of $2 n$ real equations in the $3 n$ real unknowns $z_{j}, \mu_{j}$, and so it can not determine the maximizers. The inner equal-area property adds more information. In particular, we have that for each $j$ the vector $\left(z_{j+2}-z_{j-1}\right)$ is parallel to $\left(z_{j+1}-z_{j}\right)$. So any maximizer $P^{\star}$ also satisfies the following system for suitable positive $\lambda_{j}$

$$
\left(z_{j+2}-z_{j-1}\right)=\lambda_{j}\left(z_{j+1}-z_{j}\right) .
$$

We notice that the $\lambda_{j}$ are well defined and positive because all vertices of $P^{\star}$ are distinct and $P^{\star}$ is convex.

The system (23) and the system (21) represent $4 n$ real equations in the $4 n$ real unknowns $z_{j}, \mu_{j}, \lambda_{j}$. In principle we can eliminate the unknowns $z_{j}, \mu_{j}$ from those system and reduce to $n$ equations involving only $\lambda_{j}$. This is obtained in the following theorem. 
Theorem 2.5. Let $P^{\star}$ be a maximizer of $F$ on $\mathcal{P}_{n}, n \geq 5$. Then the real numbers $\lambda_{j}$ defined by (23) satisfy

$$
\frac{\left(\lambda_{j-2}-1\right)\left(\lambda_{j-1}-1\right)}{\lambda_{j-2}}=\frac{\left(\lambda_{j+1}-1\right)\left(\lambda_{j}-1\right)}{\lambda_{j+1}},
$$

with $\lambda_{j}>1$ for $j=1,2, \ldots, n$.

Proof. From (23) we get

and

$$
z_{j+2}=z_{j-1}+\lambda_{j}\left(z_{j+1}-z_{j}\right)
$$

$$
z_{j-2}=z_{j+1}-\lambda_{j-1}\left(z_{j}-z_{j-1}\right) .
$$

By substituting in (21) and rearranging the terms we obtain

$$
\begin{gathered}
\left(z_{j+1}-z_{j}\right)\left(\mu_{j-1}+\mu_{j}+\mu_{j+1}-1-\lambda_{j} \mu_{j+1}\right)+ \\
+\left(z_{j}-z_{j-1}\right)\left(\mu_{j-1}+\mu_{j}+\mu_{j+1}-1-\lambda_{j-1} \mu_{j-1}\right)=0 .
\end{gathered}
$$

Since $P^{\star} \in \mathcal{P}_{n}$ the vertices $z_{j}$ are distinct and not collinear. This implies that the vectors $\left(z_{j+1}-z_{j}\right),\left(z_{j}-z_{j-1}\right)$ are linearly independent and their coefficients in the previous equation must be zero. Hence, we get for $j=1, \ldots, n$,

$$
\left\{\begin{array}{l}
\mu_{j-1}+\mu_{j}+\mu_{j+1}-1=\lambda_{j} \mu_{j+1} \\
\mu_{j-1}+\mu_{j}+\mu_{j+1}-1=\lambda_{j-1} \mu_{j-1}
\end{array}\right.
$$

We infer that the $\lambda_{j}$ are such that the linear system (25) has a solution $\mu_{1}, \mu_{2}, \ldots, \mu_{n}$. Taking into account the six equations involving only the five unknowns $\mu_{j-2}, \mu_{j-1}, \mu_{j}, \mu_{j+1}$, and $\mu_{j+2}$, we deduce that the determinant of the matrix

$$
\left(\begin{array}{cccccc}
1 & 1 & 1-\lambda_{j-1} & 0 & 0 & 1 \\
0 & 1 & 1 & 1-\lambda_{j} & 0 & 1 \\
0 & 0 & 1 & 1 & 1-\lambda_{j+1} & 1 \\
1-\lambda_{j-2} & 1 & 1 & 0 & 0 & 1 \\
0 & 1-\lambda_{j-1} & 1 & 1 & 0 & 1 \\
0 & 0 & 1-\lambda_{j} & 1 & 1 & 1
\end{array}\right)
$$

has to be zero. After some manipulations, we get the following equation

$$
\lambda_{j} \lambda_{j-2}\left(1-\lambda_{j+1}\right) \lambda_{j-1}\left(1-\lambda_{j}\right)-\lambda_{j-1} \lambda_{j+1}\left(1-\lambda_{j-2}\right) \lambda_{j}\left(1-\lambda_{j-1}\right)=0 .
$$

Since all $\lambda_{j}>0$, the previous equation can be simplified as in (24). Suppose that there exists a $\lambda_{j} \leq 1$. Up to an affine transformation we can assume that $z_{j-1}, z_{j}, z_{j+1}$ are three consecutive vertices of a square $Q$. Since $\lambda_{j} \leq 1, z_{j+2}$ belongs to $Q$. Then using (23) we obtain that $z_{j+3}-z_{j}$ has the direction of $z_{j+2}-z_{j+1}$. This means that $z_{j+3}$ belongs to a line supporting $Q$ at $z_{j}$. Any choice of $z_{j+3}$ on such a line gives a contradiction, since or $z_{j-1}$ belongs to the convex hull of the other vertices, or the side $z_{j+2} z_{j+3}$ intersects the boundary of $Q$.

We remark that by the definition $(23)$ of $\lambda_{j}$, we have $\lambda_{j}=d_{j} / l_{j}$ and (24) implies $P^{\star} \in \mathcal{F}_{n}$, the class of the $n$-gons with the inner-ratio property defined by (5). So from Theorem 2.5 we obtain Theorem 1.4 . 
2.2. Outer case. In order to get the Lagrange multipliers system for the outer case in a simple way we recall that $v_{j}$ is the intersection of the two lines through the consecutive vertices $z_{j-1}, z_{j}$ and the vertices $z_{j+1}, z_{j+2}$, (see Fig. 1 ), $l_{j}=\left\|z_{j+1}-z_{j}\right\|$, i.e., the length of the $j$-side, and $s_{j}=\left\|v_{j}-z_{j}\right\|, p_{j}=\left\|z_{j+1}-v_{j}\right\|$.

Proposition 2.6. Let $P^{\star}$ be a maximizer of the functional $G$ on $\mathcal{P}_{n}, n \geq 5$. Then there exist real numbers $\eta_{1}, \ldots, \eta_{n}$ such that the following equations hold for the vertices of $P^{\star}$, for $j=1, \ldots, n$.

$$
\begin{aligned}
\left(z_{j-1}-z_{j+1}\right) & =\eta_{j-2}\left(z_{j-1}-v_{j-2}\right) \frac{p_{j-2}}{l_{j-1}} \\
& +\eta_{j-1}\left(\left(v_{j-1}-z_{j-1}\right)+\left(v_{j-1}-z_{j+1}\right) \frac{p_{j-1}}{l_{j}}\right) \\
& +\eta_{j}\left(\left(z_{j+1}-v_{j}\right)+\left(z_{j-1}-v_{j}\right) \frac{s_{j}}{l_{j-1}}\right) \\
& +\eta_{j+1}\left(v_{j+1}-z_{j+1}\right) \frac{s_{j+1}}{l_{j}} .
\end{aligned}
$$

Proof. By Proposition 1.1 each maximizer $P^{\star}$ satisfies the outer equivalent triangle property. Choosing a suitable affine transformation we can assume that $\left|T_{j}(P)\right|=1$, for $j=1, \ldots, n$. By the Lagrange multipliers argument, at $P^{\star}$ the gradient of the area functional $|P|$ is a linear combination of the gradients of the constraints $\left|T_{i}(P)\right|=1$, i.e. there exist real multipliers $\eta_{j}$ such that, at $P^{\star}$,

$$
\frac{\partial|P|}{\partial \overline{z_{j}}}=\sum_{i} \eta_{i} \frac{\partial\left|T_{i}\right|}{\partial \overline{z_{j}}} \quad \text { for } \quad j=1, \ldots, n .
$$

Since $\left|T_{i}\right|$ depends only on $z_{i-1}, z_{i}, z_{i+1}, z_{i+2}$ we get

$$
\frac{4}{i} \frac{\partial\left|T_{i}\right|}{\partial \overline{z_{j}}}=0 \quad \text { for } i \notin\{j-2, j-1, j, j+1\} .
$$

By Lemma 2.3, we obtain

$$
\begin{aligned}
\frac{4}{i}\left|T_{j-2}\right|_{\overline{z_{j}}} & =\left(z_{j-1}-v_{j-2}\right) \frac{p_{j-2}}{l_{j-1}}, \\
\frac{4}{i}\left|T_{j-1}\right|_{\overline{z_{j}}} & =\left(v_{j-1}-z_{j-1}\right)+\left(v_{j-1}-z_{j+1}\right) \frac{p_{j-1}}{l_{j}}, \\
\frac{4}{i}\left|T_{j}\right|_{\overline{z_{j}}} & =\left(z_{j+1}-v_{j}\right)+\left(z_{j-1}-v_{j}\right) \frac{s_{j}}{l_{j-1}}, \\
\frac{4}{i}\left|T_{j+1}\right|_{\overline{z_{j}}} & =\left(v_{j+1}-z_{j+1}\right) \frac{s_{j+1}}{l_{j}} .
\end{aligned}
$$

The five previous equations and (22) yield (27).

Since the lengths $p_{j}, l_{j}, s_{j}$ are functions of the vertices $z_{j}$ the system (27) appears as a system of $2 n$ real equations in the unknowns $z_{j}, \eta_{j}$, i.e. $3 n$ real unknowns, and so it can not determine the maximizers $n$-gons. From the outer equivalent triangle property of any maximizer $P^{\star}$ we have that, for each $j$, the vector $\left(v_{j}-v_{j-1}\right)$ is parallel to $\left(z_{j+1}-z_{j-1}\right)$. Hence $P^{\star}$ satisfies also the following system for suitable positive $\zeta_{j}$

$$
\left(v_{j}-v_{j-1}\right)=\zeta_{j}\left(z_{j+1}-z_{j-1}\right) .
$$

We notice that the $\zeta_{j}$ are well defined and positive because all vertices of $P^{\star}$ are distinct and $P^{\star}$ is convex. 
The previous equations and system (27) represent $4 n$ real equations in the $4 n$ real unknowns $z_{j}, \eta_{j}, \zeta_{j}$. In principle we can eliminate the unknowns $z_{j}$ and $\eta_{j}$ from those systems and reduce to $n$ equations involving only $\zeta_{j}$. This is obtained in the following theorem.

Theorem 2.7. Let $P^{\star}$ be a maximizer of $G$ on $\mathcal{P}_{n}, n \geq 5$. Then the real numbers $\zeta_{j}$ defined by (28) satisfy

$$
\frac{\left(1+\zeta_{j+2}\right)}{\zeta_{j+1}\left(1+\zeta_{j+1}+\zeta_{j+2}\right)}=\frac{\left(1+\zeta_{j-1}\right)}{\zeta_{j}\left(1+\zeta_{j}+\zeta_{j-1}\right)}
$$

with $\zeta_{j}>0$, for $j=1,2, \ldots, n$.

Proof. From the outer equivalent triangle property we have that $\left(v_{j}-v_{j-1}\right)$ is parallel to $\left(z_{j+1}-z_{j-1}\right)$. From this it follows that the triangles $v_{j}, z_{j}, v_{j-1}$ and $z_{j+1}, z_{j}, z_{j-1}$ are similar. Therefore,

$$
\zeta_{j}=\frac{\left|v_{j}-v_{j-1}\right|}{\left|z_{j+1}-z_{j-1}\right|}=\frac{s_{j}}{l_{j-1}}=\frac{p_{j-1}}{l_{j}} .
$$

Moreover, from the collinearity of $v_{j}$ with $z_{j}, z_{j-1}$ we can express $v_{j}$ in terms of $z_{j}, z_{j-1}$ and positive $\zeta_{j}$

$$
v_{j}=z_{j}+\zeta_{j}\left(z_{j}-z_{j-1}\right) .
$$

Similarly we have

$$
\begin{aligned}
& v_{j+1}=z_{j+1}+\zeta_{j+1}\left(z_{j+1}-z_{j}\right), \\
& v_{j-1}=z_{j}+\zeta_{j-1}\left(z_{j}-z_{j+1}\right), \\
& v_{j-2}=z_{j-1}+\zeta_{j-1}\left(z_{j-1}-z_{j}\right) .
\end{aligned}
$$

This permits us to eliminate in (27) all the vectors $v_{i}$, i.e.,

$$
\begin{aligned}
\left(z_{j-1}-z_{j+1}\right)= & \eta_{j-2} \zeta_{j-1}^{2}\left(z_{j}-z_{j-1}\right)+ \\
& \eta_{j-1}\left[\left(z_{j}-z_{j-1}\right)+\zeta_{j}\left(z_{j}-z_{j+1}\right)+\left(z_{j}-z_{j+1}\right)\left(1+\zeta_{j}\right) \zeta_{j}\right]+ \\
& \eta_{j}\left[\left(z_{j+1}-z_{j}\right)-\zeta_{j}\left(z_{j}-z_{j-1}\right)+\left(z_{j-1}-z_{j}\right)\left(1+\zeta_{j}\right) \zeta_{j}\right]+ \\
& \eta_{j+1}\left(z_{j+1}-z_{j}\right) \zeta_{j+1}^{2} .
\end{aligned}
$$

Rearranging the terms we obtain

$$
\begin{gathered}
\left(z_{j-1}-z_{j}\right)\left(1+\eta_{j-2} \zeta_{j-1}^{2}+\eta_{j-1}-\eta_{j}\left(2 \zeta_{j}+\zeta_{j}^{2}\right)\right)+ \\
+\left(z_{j}-z_{j+1}\right)\left(1-\eta_{j-1}\left(2 \zeta_{j}+\zeta_{j}^{2}\right)+\eta_{j}+\eta_{j+1} \zeta_{j+1}^{2}\right)=0 .
\end{gathered}
$$

Since $P^{\star} \in \mathcal{P}_{n}$, the vertices $z_{j}$ are distinct and not collinear. This implies that the vectors $\left(z_{j+1}-z_{j}\right),\left(z_{j}-z_{j-1}\right)$ are linearly independent and their coefficients in the previous equation must be zero, i.e.,

$$
\left\{\begin{array}{l}
\eta_{j-2} \zeta_{j-1}^{2}+\eta_{j-1}-\eta_{j}\left(2 \zeta_{j}+\zeta_{j}^{2}\right)=-1 \\
-\eta_{j-1}\left(2 \zeta_{j}+\zeta_{j}^{2}\right)+\eta_{j}+\eta_{j+1} \zeta_{j+1}^{2}=-1
\end{array}\right.
$$


for $j=1, \ldots, n$. We shift by one the index in the first equation and adding to and subtracting from the second one, we obtain

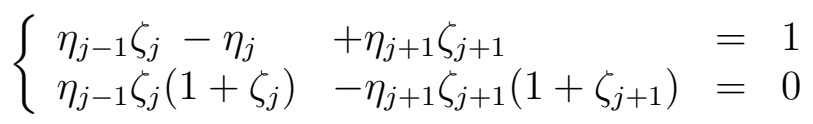

for $j=1, \ldots, n$.

We infer that the $\zeta_{j}$ are such that the linear system (33) has a solution $\eta_{1}, \eta_{2}, \ldots, \eta_{n}$. Taking into account the six equations involving only the five unknowns $\eta_{j-2}, \ldots, \eta_{j+2}$, we deduce that the determinant of the matrix

$$
\left(\begin{array}{cccccc}
\zeta_{j-1} & -1 & \zeta_{j} & 0 & 0 & 1 \\
0 & \zeta_{j} & -1 & \zeta_{j+1} & 0 & 1 \\
0 & 0 & \zeta_{j+1} & -1 & \zeta_{j+2} & 1 \\
\zeta_{j-1}+\zeta_{j-1}^{2} & 0 & -\zeta_{j}-\zeta_{j}^{2} & 0 & 0 & 0 \\
0 & \zeta_{j}+\zeta_{j}^{2} & 0 & -\zeta_{j+1}-\zeta_{j+1}^{2} & 0 & 0 \\
0 & 0 & \zeta_{j+1}+\zeta_{j+1}^{2} & 0 & -\zeta_{j+2}-\zeta_{j+2}^{2} & 0
\end{array}\right)
$$

has to be zero. After some manipulations we obtain

$$
\begin{gathered}
\zeta_{j-1}\left(1+\zeta_{j}\right)\left(1+\zeta_{j+1}\right) \zeta_{j+2} \cdot \\
\cdot\left[\zeta_{j}\left(1+\zeta_{j+2}\right)\left(1+\zeta_{j}+\zeta_{j-1}\right)-\zeta_{j+1}\left(1+\zeta_{j-1}\right)\left(1+\zeta_{j+1}+\zeta_{j+2}\right)\right]=0 .
\end{gathered}
$$

Since $\zeta_{j}>0$, the previous equation can be simplified as in the statement (29) and we conclude the proof.

We remark that by (30) the relations between the $\zeta_{j}$ 's of Theorem 2.7 can be expressed in terms of ratios of the length of the sides of the outer triangles $W_{j}\left(P^{\star}\right)$. It turns out that (29) implies that $P^{\star} \in \mathcal{G}_{n}$, the class of the $n$-gons with the outer ratio property defined by (6). So from Theorem 2.7 we obtain Theorem 1.5.

\section{Circulant Systems}

In this section we have collected the steps of the proofs which are not strictly related with the geometry of the problem. We focus on the systems (24), (29) and we prove that the only suitable solutions are those independent of $j$.

First we deal with the inner problem.

Proposition 3.1. Any solution of the system of equations

$$
\frac{\left(\lambda_{j-2}-1\right)\left(\lambda_{j-1}-1\right)}{\lambda_{j-2}}=\frac{\left(\lambda_{j+1}-1\right)\left(\lambda_{j}-1\right)}{\lambda_{j+1}},
$$

for $j=1,2, \ldots, n$, with $\lambda_{j}>1$ for all $j$, satisfies $\lambda_{i}=\lambda_{j}$ for all $i$ and $j$.

Proof. Set $\gamma_{j}=1 /\left(\lambda_{j}-1\right)$, for all $j$. Notice that $\lambda_{j}>1$ implies $\gamma_{j}>0$. Then equations (24) reduce to

$$
\gamma_{j-1}\left(1+\gamma_{j-2}\right)=\gamma_{j}\left(1+\gamma_{j+1}\right)
$$


Consider the maximum number among the expressions $\gamma_{j}\left(1+\gamma_{j-1}\right)$, for all $j$. Assume this maximum is attained by $\gamma_{M}\left(1+\gamma_{M-1}\right)$. From (34) we obtain

$$
\gamma_{M}\left(1+\gamma_{M-1}\right) \geq \gamma_{j}\left(1+\gamma_{j+1}\right)
$$

for every $j=1,2, \ldots, n$. Now, from $\gamma_{M}\left(1+\gamma_{M-1}\right) \geq \gamma_{M-1}\left(1+\gamma_{M}\right)$ we have

$$
\gamma_{M} \geq \gamma_{M-1} \text {. }
$$

From $\gamma_{M}\left(1+\gamma_{M-1}\right) \geq \gamma_{M}\left(1+\gamma_{M+1}\right)$ we find

$$
\gamma_{M-1} \geq \gamma_{M+1}
$$

From (34) we deduce that $\gamma_{M}\left(1+\gamma_{M-1}\right)=\gamma_{M+1}\left(1+\gamma_{M+2}\right)$ and so we can also write $\gamma_{M+1}(1+$ $\left.\gamma_{M+2}\right) \geq \gamma_{M+1}\left(1+\gamma_{M}\right)$, which implies that

$$
\gamma_{M+2} \geq \gamma_{M}
$$

and $\gamma_{M+1}\left(1+\gamma_{M+2}\right) \geq \gamma_{M+2}\left(1+\gamma_{M+1}\right)$, which yields

$$
\gamma_{M+1} \geq \gamma_{M+2} .
$$

By combining inequalities (35), (36), (37), and (38) we discover $\gamma_{M+1} \geq \gamma_{M+2} \geq \gamma_{M} \geq \gamma_{M-1} \geq$ $\gamma_{M+1}$ and so $\gamma_{M-1}=\gamma_{M}=\gamma_{M+1}=\gamma_{M+2}$. Taking into account (34) again yields $\gamma_{i}=\gamma_{j}$, for all $i$ and $j$.

We now turn to the outer problem.

Proposition 3.2. Any solution of the system of equations

$$
\frac{\zeta_{j}\left(1+\zeta_{j+2}\right)}{1+\zeta_{j+1}+\zeta_{j+2}}=\frac{\zeta_{j+1}\left(1+\zeta_{j-1}\right)}{1+\zeta_{j}+\zeta_{j-1}}
$$

for $j=1,2, \ldots, n$, with $\zeta_{j}>0$ for all $j$, satisfies $\zeta_{i}=\zeta_{j}$ for all $i$ and $j$.

Proof. Set

$$
a_{j}=\frac{\zeta_{j}}{1+\zeta_{j}+\zeta_{j+1}}, \quad b_{j}=\frac{\zeta_{j+1}}{1+\zeta_{j}+\zeta_{j+1}} .
$$

Notice that $\zeta_{j}>0$, for all $j$, implies that all $a_{j}$ and $b_{j}$ are positive and that

$$
a_{j}+b_{j}<1 \text {. }
$$

The system (29) can be rewritten in terms of $a_{j}, b_{j}$ as

$$
\frac{1-a_{j+1}}{b_{j}}=\frac{1-b_{j-1}}{a_{j}} .
$$

Furthermore, it is easy to check that the relation

$$
\frac{1-a_{j}}{b_{j}}=\frac{1-b_{j+1}}{a_{j+1}}
$$

also holds for every $j=1,2, \ldots, n$. 
Now consider the maximum number among the expressions $\left(1-a_{j+1}\right) / b_{j}$ or $\left(1-a_{j}\right) / b_{j}$ involved in (41) and (42) and call it $C$. Assume first that $C$ appears in (41), i.e., $C=\frac{1-a_{M+1}}{b_{M}}$, for some $M$. From $\frac{1-a_{M+1}}{b_{M}} \geq \frac{1-a_{M}}{b_{M}}$ we infer

$$
a_{M} \geq a_{M+1} .
$$

From

we deduce

$$
\frac{1-b_{M-1}}{a_{M}}=\frac{1-a_{M+1}}{b_{M}} \geq \frac{1-b_{M}}{a_{M}}
$$

$$
b_{M} \geq b_{M-1} .
$$

From

$$
\frac{1-a_{M+1}}{b_{M}}=\frac{1-b_{M-1}}{a_{M}} \geq \frac{1-a_{M}}{b_{M-1}}
$$

we deduce $b_{M-1}-b_{M-1}^{2} \geq a_{M}-a_{M}^{2}$ and then

$$
\left(b_{M-1}-a_{M}\right)\left(1-b_{M-1}-a_{M}\right) \geq 0 .
$$

Since $b_{M-1} \leq b_{M}$ by (44), inequality (40) implies that $\left(1-b_{M-1}-a_{M}\right)>0$. Therefore

$$
b_{M-1} \geq a_{M} .
$$

Analogously, from

we infer

$$
\frac{1-a_{M+1}}{b_{M}} \geq \frac{1-a_{M+2}}{b_{M+1}}=\frac{1-b_{M}}{a_{M+1}}
$$

$$
\left(a_{M+1}-b_{M}\right)\left(1-a_{M+1}-b_{M}\right) \geq 0 .
$$

Inequalities (40), (43) ensure that $\left(1-a_{M+1}-b_{M}\right)>0$ and so

$$
a_{M+1} \geq b_{M} \text {. }
$$

Combining inequalities (43), (44), (45), (46) we obtain

$$
b_{M-1} \geq a_{M} \geq a_{M+1} \geq b_{M} \geq b_{M-1}
$$

and so

$$
b_{M-1}=a_{M}=a_{M+1}=b_{M} .
$$

Now it is easy to use (41) and (42) to deduce that all $a_{j}$ and $b_{j}$ have to be equal, and from this fact and (39) that all the $\zeta_{j}$ are equal.

Assume now that $C$ appears in (42), i.e., $C=\left(1-a_{M}\right) / b_{M}$, for some $M$. From $\left(1-a_{M}\right) / b_{M} \geq$ $\left(1-b_{M}\right) / a_{M}$ and (40) we deduce

$$
a_{M} \geq b_{M} .
$$

From $\frac{1-b_{M+1}}{a_{M+1}}=\frac{1-a_{M}}{b_{M}} \geq \frac{1-a_{M+1}}{b_{M+1}}$ and (40) we deduce

$$
b_{M+1} \geq a_{M+1} .
$$


From $\frac{1-b_{M+1}}{a_{M+1}}=\frac{1-a_{M}}{b_{M}} \geq \frac{1-b_{M}}{a_{M+1}}$ we infer

$$
b_{M} \geq b_{M+1} .
$$

From $\frac{1-a_{M}}{b_{M}} \geq \frac{1-a_{M+1}}{b_{M}}$ we obtain

$$
a_{M+1} \geq a_{M} .
$$

Once again we have obtained a sequence of inequalities that can be satisfied only if $a_{M}=$ $a_{M+1}=b_{M}=b_{M+1}$. As in the previous case, it is now easy to conclude that all $\zeta_{j}$ have to be equal.

G. Ottaviani has analyzed the case $n=7$ for the inner and outer problem. By using the computer algebra system Macaulay, he found that in the complex variables $\gamma_{1}, \ldots, \gamma_{7}$, the system (34) has a zero set which consists of the trivial line $\gamma_{1}=\cdots=\gamma_{7}$ and of an algebraic surface of degree 14. It is interesting that the line and the surface are disjoint components. Similarly, he found that for the outer problem the zero set of the system (29) contains the trivial line and a surface of degree 71.

\section{Proofs of Theorems 1.6 And 1.7}

In this section we complete the characterization of the maximizers of $F$ and $G$, thus proving Theorems 1.6, 1.7. To do this we turn back to geometry.

Proof of Theorem 1.6. As noted in Section 2.1, each polygon $P$ contained in $\Phi_{n} \cap \mathcal{F}_{n}$ satisfies systems (23), (24). By Proposition 3.1 we deduce that there exists a $\lambda>1$ such that

$$
z_{j+2}-z_{j-1}=\lambda\left(z_{j+1}-z_{j}\right), \text { for all } j .
$$

Such a condition is satisfied only by affinely regular $n$-gons. This is proved in $[4$, Statement 3 , Theorem 1], where the result is attributed to Coxeter [3]. We provide a proof for completeness and for the convenience of the reader.

Up to an affine transformation, we can assume that three consecutive edges of $P, z_{1} z_{2}, z_{2} z_{3}$ and $z_{3} z_{4}$, say, all have length one. By (51), the quadrilateral $z_{1} z_{2} z_{3} z_{4}$ is an isosceles trapezium, and so the angles of $P$ in $z_{2}$ and $z_{3}$ are also equal. Denote by $r_{2}$ the bisector of the angle in $z_{2}$. By assumption, $r_{2}$ is the axis of the segment $z_{1} z_{3}$. Since the vertex $z_{n}$, consecutive to $z_{1}$, is determined by the relation $z_{n}-z_{3}=\lambda\left(z_{1}-z_{2}\right)$ and $z_{4}$ by $z_{4}-z_{1}=\lambda\left(z_{3}-z_{2}\right)$, it is easily seen that $r_{2}$ is the axis of the segment $z_{4} z_{n}$. Similarly, $z_{n-1}$ and $z_{5}$ are uniquely determined by the previous vertices (and $\lambda$ ) and then $r_{2}$ is also the axis of the segment $z_{5} z_{n-1}$. This argument shows that $r_{2}$ is a symmetry axis of $P$. Analogously, considering the vertex $z_{3}$ of the trapezium $z_{1} z_{2} z_{3} z_{4}$, the bisector $r_{3}$ of the angle in $z_{3}$ is a symmetry axis of $P$. This clearly implies that $P$ is a regular polygon.

Proof of Theorem 1.7. As noted in Section 2.2, each polygon $P$ contained in $\Gamma_{n} \cap \mathcal{G}_{n}$ satisfies systems (28), (29). By Proposition 3.2 we deduce that there exists a $\zeta>0$ such that

$$
v_{j}-v_{j-1}=\zeta\left(z_{j+1}-z_{j-1}\right), \text { for all } \mathrm{j} .
$$


This means that the pairs of triangles $v_{j-1} v_{j} z_{j}, z_{j+1} z_{j-1} z_{j}$ are all similar with the same ratio of similarity. Hence,

$$
\begin{aligned}
v_{j}-z_{j} & =\zeta\left(z_{j}-z_{j-1}\right), \\
v_{j}-z_{j+1} & =\zeta\left(z_{j+1}-z_{j+2}\right) .
\end{aligned}
$$

Therefore the triangles $v_{j} z_{j+1} z_{j}, v_{j} z_{j+2} z_{j-1}$ are also similar and

$$
z_{j+2}-z_{j-1}=(1+\zeta)\left(z_{j+1}-z_{j}\right), \text { for all } j .
$$

Such a condition is just like (51) and the same argument of the previous proof implies that $P$ is an affinely regular polygon.

Theorems 1.6 and 1.7 were the last ingredients in the proof of Theorem 1.8, whose quantitative form is the following.

Theorem 4.1. Let $P \in \mathcal{P}_{n}, n \geq 5$, then

$$
\begin{gathered}
\min _{j=1, \ldots, n}\left|T_{j}(P)\right| \leq|P| \frac{2 \sin ^{2} \pi / n}{n \cos 2 \pi / n}, \\
\min _{j=1, \ldots, n}\left|W_{j}(P)\right| \leq|P| \frac{4 \sin ^{2}(\pi / n)}{n},
\end{gathered}
$$

and equality holds if and only if $P$ is an affinely regular $n$-gon.

Notice that (54) implies the inequality [16, Lemma 3].

\section{EXTENSIONS AND AFFINE LENGTH}

Let $C$ be a planar convex body, and let $\gamma$ be a connected closed proper subset of $\partial C$, i.e., a closed arc with endpoints $a$ and $b$. Define $C(\gamma)$ as the intersection of all half-planes supporting $C$ at boundary points in $\partial C \backslash \gamma$. The boundary of $C(\gamma)$ is obtained by extending $\partial C \backslash \gamma$ with the continuations of the half-lines tangent in $a$ and $b$ at $\partial C \backslash \gamma$. Consider the region $I(C, \gamma)=C(\gamma) \backslash C$ and the area ratio $|I(C, \gamma)| /|C|$. Now let $\Gamma$ be a finite family of connected closed subsets $\gamma_{i}$ of $\partial C$ with disjoint empty interior:

$$
\Gamma=\left\{\gamma_{1}, \ldots, \gamma_{n}: \quad \gamma_{i} \subset \partial C, \operatorname{int}\left(\gamma_{i}\right) \cap \operatorname{int}\left(\gamma_{j}\right)=\emptyset \text { for } i \neq j, \quad i, j=1, \ldots, n\right\}
$$

Define

$$
G(C, \Gamma)=\min _{j=1, \ldots, n} \frac{\left|I\left(C, \gamma_{j}\right)\right|}{|C|} .
$$

Denoting by $\# \Gamma$ the number of elements of $\Gamma$, we define

$$
G_{n}(C)=\max _{\# \Gamma=n} G(C, \Gamma) .
$$

Theorem 5.1. For $n>4$ and for any planar convex body $C$ we have

$$
G_{n}(C) \leq \max _{P \in \mathcal{P}_{n}} G(P) .
$$

Equality holds if and only if $C$ is an affinely regular $n-g o n$. 
Proof. Let $C$ be a planar convex body and let $\Gamma$ be a finite family of arcs $\gamma_{i}$ on $\partial C$ with endpoints $a_{i}, b_{i}$. We can consider families of arcs which partition $\partial C$, i.e., such that $a_{i+1}=b_{i}$ for all $i$. This can be proved by taking a partition $\Gamma^{\star}$ with $\operatorname{arcs} \gamma_{i}^{\star}$ so that $\gamma_{i} \subset \gamma_{i}^{\star}$. Since $I\left(C, \gamma_{i}\right) \subset I\left(C, \gamma_{i}^{\star}\right)$ we have $G(C, \Gamma) \leq G\left(C, \Gamma^{\star}\right)$.

Let $P$ be the convex polygon whose vertices are the endpoints $a_{i}, b_{i}$ of the $\operatorname{arcs} \gamma_{i}$ of $\Gamma$. Clearly, $P \subset C$ and $P$ has exactly $n$ edges. Moreover, if $T_{i}(P)$ is the outer triangle defined in the introduction corresponding to the edge with vertices $a_{i}$, $b_{i}$, then $I\left(C, \gamma_{i}\right) \subset T_{i}(P)$, and

$$
G(C, \Gamma) \leq G(P) .
$$

This immediately yields (58) and the equality conditions.

Corollary 5.2. Let $K$ and $K^{\prime}$ be two planar convex bodies such that their symmetric difference has $n>4$ connected components $C_{1}, \ldots, C_{n}$. Then

$$
\min _{i=1, \ldots, n}\left|C_{i}\right| \leq\left|K \cap K^{\prime}\right| \max _{P \in \mathcal{P}_{n}} G(P) .
$$

Equality holds if and only if $K \cap K^{\prime}$ is an affinely regular polygon with $n=2 m$ edges and, up to an affine transformation, $K$ and $K^{\prime}$ are two congruent regular $m$-gons, and $K^{\prime}$ is $K$ rotated by $\pi / m$ about its center.

Proof. We consider the family $\Gamma$ of the closed arcs

$$
\gamma_{i}=\partial C_{i} \cap \partial\left(K \cap K^{\prime}\right) \text {. }
$$

Since

$$
\left|C_{i}\right| \leq\left|I\left(K \cap K^{\prime}, \gamma_{i}\right)\right|
$$

we can apply the previous theorem to $C=K \cap K^{\prime}$. Equality holds if and only if $C$ is an affinely regular $2 m$-gon and so $K$ and $K^{\prime}$ have to be affinely regular $m$-gons.

We present another possible extension of the previous area functionals. Let $C$ be a convex body. Let $D$ be a convex body containing $C$ and such that $D \backslash C$ consists of at least $m$ connected components $D_{1}, \ldots, D_{m}$. Let

$$
H_{m}(C, D)=\min _{i=1, \ldots, m}\left|D_{i}\right| .
$$

Arguing as above, when we look for the maximizers of $H_{m}(C, D)|C|^{-1}$ it is easy to see that one can assume that $C$ and $D$ are $m$-gons. The number $\#(D \backslash C)$ of the connected components of $D \backslash C$ is $m$, and hence the edges of $D$ support $C$ at its vertices. We get the following result.

Theorem 5.3. For $m \geq 3$ and for any two planar convex bodies $C, D$ with $C \subset D, \#(D \backslash C)=$ $m$, we have

$$
\frac{H_{m}(C, D)}{|C|} \leq \frac{1}{m} \tan ^{2} \frac{\pi}{m}
$$

Equality holds if and only if $C, D$ are affinely regular $m$-gons and the vertices of $C$ are the midpoints of the edges of $S$. 
Proof. Standard compactness arguments show that $H_{m}(C, D)|C|^{-1}$ has a maximum. As already stated in advance, we can assume that such a maximum is attained when $C$ and $D$ are two $m$-gons $P$ and $S, P \subset S$. Let $z_{j}$ be the vertices of $P$ and $v_{j}$ the vertices of $S$ labeled so that $z_{j}$ belongs to the segment $v_{j} v_{j-1}$.

First we prove that all triangles $S_{j}$ with vertices $z_{j}, v_{j}, z_{j+1}$ have equal area. Indeed, if $\left|S_{i}\right|>\min _{j=1, \ldots, m}\left|S_{j}\right|$, then a small counterclockwise rotation of the side of $S$ through $z_{i}$ around $z_{i}$ decreases the area of $S_{i}$ and increases the area of $S_{i-1}$. Possible iterations of this procedure to different edges of $S$ would permit to increase the value of our functional. The assumption on the maximality of the pair $(P, S)$ implies

$$
\left|S_{i}\right|=\left|S_{i-1}\right| \text {, }
$$

Arguing as in the proof of Proposition 2.4, we consider the Lagrange multipliers system relative to $|P|$ as a function of $z_{j}, v_{j}$, under the constraints $\left|S_{j}\right| \geq$ const, $z_{j} \in v_{j-1} v_{j}$. Denote by $\mu_{j}$ the parameters corresponding to the constraints $\left|S_{j}\right| \geq$ const, and by $\nu_{j}$ the one corresponding to $\operatorname{det}\left(z_{j}-v_{j-1}, z_{j}-v_{j}\right)=A_{i}=0$. Hence, we obtain the system

$$
\begin{gathered}
\frac{\partial|P|}{\partial \overline{z_{j}}}=\sum_{i=1}^{m} \mu_{i} \frac{\partial\left|S_{i}\right|}{\partial \overline{z_{j}}}+\sum_{i=1}^{m} \nu_{i} \frac{\partial A_{i}}{\partial \overline{z_{j}}} \quad \text { for } j=1, \ldots, n . \\
0=\frac{\partial|P|}{\partial \overline{v_{j}}}=\sum_{i=1}^{m} \mu_{i} \frac{\partial\left|S_{i}\right|}{\partial \overline{v_{j}}}+\sum_{i=1}^{m} \nu_{i} \frac{\partial A_{i}}{\partial \overline{v_{j}}} \quad \text { for } j=1, \ldots, n .
\end{gathered}
$$

Since we are looking for minimizers of $|P|$ with unilateral constraints $\left|S_{j}\right| \geq$ const, we have

$$
\mu_{j} \geq 0 \text { for } j=1, \ldots, n .
$$

Taking into account the results in Section 2 we get:

$$
\begin{gathered}
\left(z_{j-1}-z_{j+1}\right)=\mu_{j-1}\left(v_{j-1}-z_{j-1}\right)+\mu_{j}\left(z_{j+1}-v_{j}\right)+\nu_{j}\left(v_{j-1}-v_{j}\right), \\
0=\mu_{j}\left(z_{j}-z_{j+1}\right)+\nu_{j}\left(z_{j}-v_{j-1}\right)+\nu_{j+1}\left(v_{j+1}-z_{j+1}\right) .
\end{gathered}
$$

The constrain $A_{i}=0$ can be written as $z_{j}=v_{j-1}+\rho_{j}\left(v_{j}-v_{j-1}\right)$, where the variables $\rho_{j}$ satisfy $0<\rho_{j}<1$. Consequently,

$$
\left|S_{j}\right|=\left|\left(z_{j+1}-v_{j}\right) \wedge\left(z_{j}-v_{j}\right)\right|=\rho_{j+1}\left(1-\rho_{j}\right)\left|U_{j}\right|,
$$

where $U_{j}$ denotes the triangle $v_{j-1} v_{j} v_{j+1}$. Identity (61) yields

$$
\rho_{j+1}\left(1-\rho_{j}\right)\left|U_{j}\right|=\rho_{j}\left(1-\rho_{j-1}\right)\left|U_{j-1}\right| .
$$

Equations (63) and (64) become

$$
\begin{gathered}
\left(1+\mu_{j-1}\right)\left(1-\rho_{j-1}\right)\left(v_{j-2}-v_{j-1}\right)+\left(1+\mu_{j}\right) \rho_{j+1}\left(v_{j}-v_{j+1}\right)+\left(v_{j-1}-v_{j}\right)\left(1-\nu_{j}\right)=0 \\
0=\left(v_{j}-v_{j-1}\right)\left(\rho_{j}\left(\mu_{j}+\nu_{j}\right)-\mu_{j}\right)+\left(v_{j+1}-v_{j}\right)\left(-\rho_{j+1}\left(\mu_{j}+\nu_{j+1}\right)+\nu_{j+1}\right) .
\end{gathered}
$$

Since $\left(v_{j}-v_{j-1}\right)$ and $\left(v_{j+1}-v_{j}\right)$ are linearly independent, we obtain

$$
\mu_{j}\left(1-\rho_{j}\right)=\rho_{j} \nu_{j},
$$




$$
\mu_{j} \rho_{j+1}=\left(1-\rho_{j+1}\right) \nu_{j+1} .
$$

Taking the cross product of $(66)$ with the vector $\left(v_{j}-v_{j-1}\right)$ yields

$$
\left(1+\mu_{j-1}\right)\left(1-\rho_{j-1}\right) U_{j-1}-\left(1+\mu_{j}\right) \rho_{j+1} U_{j}=0 .
$$

By means of (65) the latter reduces to

$$
\left(1+\mu_{j}\right) \rho_{j}=\left(1+\mu_{j-1}\right)\left(1-\rho_{j}\right) .
$$

The systems (68), (69), (70) are $3 n$ real equations in $3 n$ real unknowns $\mu_{j}, \rho_{j}, \nu_{j}$. From the first two equations we get

$$
\mu_{j}\left(1-\rho_{j}\right)^{2}=\mu_{j-1} \rho_{j}^{2}
$$

A comparison with (70) implies

$$
\left(1+\mu_{j}\right)^{2} \mu_{j}=\left(1+\mu_{j-1}\right)^{2} \mu_{j-1} .
$$

Since the function $y=(1+x)^{2} x$ is injective for $y>0$, inequality (62) implies that $\mu_{j}=\mu$, for all $j$.

From (70) we deduce $\rho_{j}=1 / 2$ and then, from (68), that $\nu_{j}=\mu$, for all $j$. Hence, equation (66) gives

$$
(1+\mu) v_{j-2}-2 \mu v_{j-1}+2 \mu v_{j}-(1+\mu) v_{j+1}=0,
$$

which implies that $v_{j-2} v_{j+1}$ is parallel to $v_{j-1} v_{j}$ and their ratio is independent of $j$.

As in the proof of Theorem 1.6(see (51)), we deduce that $S$ is affinely regular.

Given a planar convex body $K$ and a natural number $n \geq 3$, we denote by $\mathcal{P}_{n}^{i}(K)$ and $\mathcal{P}_{n}^{c}(K)$ the class of $n$-gons inscribed in $K$ or containing $K$, respectively. For any $n$ distinct points $z_{i} \in \partial K$ we consider the polygon $P=\operatorname{conv}\left\{z_{1}, \ldots, z_{n}\right\} \in \mathcal{P}_{n}^{i}(K)$ and lines $s_{i}$ supporting $K$ at the vertices $z_{i}$ of $P$. Let $S_{i}(K)$ be the triangle bounded by the lines $s_{i-1}, s_{i}$ and the side at $z_{i-1} z_{i}$, and $S$ be the polygon bounded by the lines $s_{i}$. Hence, $S$ is a $n$-gon in $\mathcal{P}_{n}^{c}(K)$. This is equivalent to choosing two $n$-gons $P, S$, with $P \in \mathcal{P}_{n}^{i}(K), S \in \mathcal{P}_{n}^{c}(K) \cap \mathcal{P}_{n}^{c}(P)$. In this case, for brevity, we say that the pair $(P, S)$ belongs to $\mathcal{P}_{n}^{i, c}(K)$. Define

$$
A L_{n}(K)=2 n \max \left\{\min _{j=1, \ldots, n}\left|S_{j}(K)\right|^{\frac{1}{3}}:(P, S) \in \mathcal{P}_{n}^{i, c}(K)\right\} .
$$

Since $n \geq 3, A L_{n}(K)$ is finite for every $K$. The symbol we chose to denote these functionals is justified by some properties listed below, which present $A L_{n}$ as a discretization of the affine length of the boundary of $K$. Recalling the functional $H_{n}(P, S)$ introduced above, we have

$$
A L_{n}(K)=2 n \max \left\{H_{n}(P, S)^{\frac{1}{3}}:(P, S) \in \mathcal{P}_{n}^{i, c}(K)\right\} .
$$

As in the proof of Theorem 5.3 it turns out that the previous maximum is attained when all the $S_{i}(K)$ have the same area. In the sequel we denote by $\mathcal{E}_{n}$ the class of pairs of $n$-gons with the property that all the $S_{i}(K)$ have equal area.

Proposition 5.4. For every planar convex body $K$ and $n \geq 3$,

$$
A L_{n}(K)=2 n \max \left\{H_{n}(P, S)^{\frac{1}{3}}:(P, S) \in \mathcal{P}_{n}^{i, c}(K) \cap \mathcal{E}_{n}\right\} .
$$


Proof. Clearly, $\left|S_{j}(K)\right|$ is a continuous function of $z_{i}$ and $s_{i}$ for all $i$ and $j$. If $z_{i}$ moves towards $z_{i+1}$, then $\left|S_{i+1}(P)\right|$ decreases and $\left|S_{i}(P)\right|$ increases, unless one or both of them remain equal to zero. Furthermore, if $s_{i}$ rotates around $z_{i}$ counterclockwise, then $\left|S_{i+1}(P)\right|$ decreases and $\left|S_{i}(P)\right|$ increases. These facts easily imply that, if $(P, S)$ does not belong to $\mathcal{E}_{n}$, then we can move the supporting lines $s_{i}$ together with the points $z_{i}$ increasing all $\left|S_{j}(K)\right|$.

By Proposition 5.4 the functional $A L_{n}$ is then related to the arithmetic average of the cubic root of $\left|S_{j}(K)\right|$, i.e.,

$$
A L_{n}(K)=2 \max _{(P, S) \in \mathcal{P}_{n}^{i, c}(K) \cap \mathcal{E}_{n}} \sum_{j=1}^{n}\left|S_{j}(K)\right|^{\frac{1}{3}} .
$$

This further clarifies the connection with the affine length of $K, \Omega_{1}(K)$, as defined, for example, by Ludwig in [17, Section 3]. There Ludwig proved that all upper (or lower) semicontinuous and equi-affine invariant valuations on the space of planar compact convex sets (endowed with the Hausdorff metric) are linear combinations of three basic valuations: the Euler characteristic, the area, and the affine length.

Such a characterization is the main ingredient in the proof of the following.

Proposition 5.5. If $K$ is a planar convex body, then

$$
\inf _{n \in \mathbb{N}} A L_{n}(K)=\Omega_{1}(K) .
$$

We notice that $A L_{n}(K)=0$ when $K$ is a polygon with less than $n$ vertices. Moreover, these are the only convex sets where $A L_{n}$ vanishes.

Proof. Set $A L(K)=\inf _{n} A L_{n}(K)$. Since the functionals $A L_{n}$ are continuous and equi-affine invariant, it is easy to verify that $A L$ is equi-affine invariant and upper semicontinuous. Following the arguments used by Ludwig in [17, Theorem 2], it can be proved that $A L$ is a valuation, i.e.,

$$
A L(H \cup K)+A L(H \cap K)=A L(H)+A L(K),
$$

for every pair of convex bodies such that $H \cup K$ is convex. By [17, Theorem 1], it follows that $A L$ is a linear combination of the affine length, the area, and the Euler characteristic. Since $A L_{n}(P)$ vanishes when $P$ is a polygon with less than $n$ vertices, $A L$ has to be a multiple of the affine length. A simple calculation of $A L$ at the unit disc yields the result.

We present now some straightforward consequences of Theorem 5.3.

Theorem 5.6. If $K$ is a planar convex body and $n \geq 3$, then

$$
A L_{n}(K) \leq 2\left(n \tan \frac{\pi}{n}\right)^{\frac{2}{3}} \max _{P \in \mathcal{P}_{n}^{i}(K)}|P|^{\frac{1}{3}}
$$

and equality holds if and only if exists an affinely regular $n$-gon of maximal area in $\mathcal{P}_{n}^{i}(K)$.

Corollary 5.7. If $K$ is a planar convex body and $n \geq 3$, then

$$
A L_{n}(K) \leq 2\left(n \tan \frac{\pi}{n}\right)^{\frac{2}{3}}|K|^{\frac{1}{3}}
$$


and equality holds if and only if $K$ is an affinely regular $n$-gon.

Corollary 5.7 and Proposition 5.5 yield the affine isoperimetric inequality

$$
\Omega_{1}(K) \leq 2 \pi^{\frac{2}{3}}|K|^{\frac{1}{3}}
$$

without the equality conditions, which are known to characterize ellipses. Notice that equality in the formula of Theorem 5.6 implies that $K$ has at least $n$ points of intersection with a suitable ellipse, and so its Hausdorff distance from that ellipse decreases as $n$ increases.

It is well known that the affine length of a planar convex body $K$ is strictly related to the approximation of $K$ by polygons. We refer the interested reader to [8] for an extensive review. Theorems 5.6 implies that, for any planar convex body $K$ and $n \geq 3$, there exists a polygon $P \in \mathcal{P}_{n}^{i}(K)$ such that

$$
|P| \geq \frac{A L_{n}(K)^{3}}{8 n^{2} \tan ^{2}\left(\frac{\pi}{n}\right)} .
$$

Proposition 5.5 permits to replace $A L_{n}(K)$ with $\Omega_{1}(K)$ in the previous inequality. This yields a sharp lower bound for the approximation of $K$ with polygons from $\mathcal{P}_{n}^{i}(K)$, which follows also from the affine isoperimetric inequality and a result of Blaschke (see [8, Section 4]).

\section{Applications to tomography}

The following basic example is shown in [7]. Consider a regular $n$-gon $Q$ centered at a fixed point $o$, and its rotation $Q^{\prime}$ by $\pi / n$ about $o$. The convex hull of $Q$ and $Q^{\prime}$ is a $2 n$-gon; let $\theta$ be a direction parallel to one of its edges. It is easy to see that $Q$ and $Q^{\prime}$ have the same parallel $\mathrm{X}$-rays in the direction $\theta$, i.e., any line $l_{\theta}$ parallel to $\theta$ intersects $Q$ in a segment (possibly empty), which has the same length as the intersection of $l_{\theta}$ with $Q^{\prime}$. Note that this property is affinely invariant. This example arises in many papers, mainly related to Geometric and Discrete Tomography. We refer the interested reader to [6] and [12].

To clarify the connection of the previous sections with tomography we need to give a more precise definition of an X-ray.

Given a direction $\theta$, the line through $o$ orthogonal to $\theta$ is denoted by $\theta^{\perp}$. Given a convex body $H$ consider the intersection of $H$ with any line $l_{\theta}$ parallel to $\theta$ through a point $p$ on $\theta^{\perp}$. The one-dimensional measure of this intersection, as a function of $p$, is called the parallel X-ray of $H$ in the direction $\theta$.

The above example of $Q$ and $Q^{\prime}$ can be interpreted in the following way: There exist two convex bodies having the same X-rays in $n$ mutually non-parallel directions, namely with respect to directions parallel to $n$ consecutive edges of the convex hull of $Q$ and $Q^{\prime}$. Since this is invariant under affine transformations, a more general family of such situations can occur. Gardner and McMullen [5] prove that convex bodies are determined by X-rays taken in any set of directions that is not a subset of the directions of the edges of an affinely regular polygon. This result gives a solution to the problem of the unique determination for the X-ray problem proposed by Hammer [9]: How many parallel X-ray pictures of a convex body must be taken in order to permit its exact reconstruction? 
In fact, for example, any set of four directions whose slopes have a transcendental cross ratio solves Hammer's problem. Hammer's problem can be seen as a particular case of the more general inverse problem of reconstruction of a homogeneous planar body from tomographic data. Volčič shows in [19] that the reconstruction of $H$ is well posed when the set of directions guarantees uniqueness. Roughly speaking, if we know all the X-rays of $H$ in such directions, and these X-rays contain errors $\varepsilon$ as small as we want, then the corresponding reconstructions $H_{\varepsilon}$ converge to $H$ when $\varepsilon$ goes to zero. Consider the case when a finite number $n$ of X-rays of $H$ are exactly known, but the directions are determined up to an error $\delta$. The error $\delta$ has to be small enough to distinguish the given $n$ directions among them. For any positive $\delta$, we cannot distinguish the set $S$ of the given directions from the sets of non-uniqueness in the Gardner-McMullen theorem. Therefore, the results of well-posedness proved by Volčič [19] can not be used. However, in [13] the following result is proved:

$$
\left|K \triangle K^{\prime}\right| \leq l^{2}(8 n)^{-1} \tan \frac{\pi}{n}
$$

where $l$ is the length of the boundary of $K \cap K^{\prime}$ and $K \triangle K^{\prime}$ is the symmetric difference. Inequality (73) can be seen as a stability result and is optimal not only in the order but also in the constant, since equality holds if and only if the $n$ directions, $K$ and $K^{\prime}$ are chosen as in the example at the beginning of this section. Inequality (73) is not affine invariant, while Hammer's problem is. An affine-invariant inequality in which $l^{2}$ is replaced by $\left|K \cap K^{\prime}\right|$ is proved in [14] for sets of three directions. The idea of the proof of (73) and in [14] is the following:

Let $R$ be a connected component of $K \triangle K^{\prime}$. For every direction $\theta \in S$, let $\theta R$ be the connected component of $K \triangle K^{\prime}$ different from $R$ with the same X-ray of $R$ in the direction $\theta$. Let

$$
W(R)=\bigcup_{h \in \mathbb{N}, \theta_{i_{j}} \in S} \theta_{i_{h}} \cdots \theta_{i_{1}} R
$$

be the system of components associated to $R$. In [13, Proposition 2] (see also [6, Lemma 1.2.6]) it is proved that $W(R)$ consists of a finite number $h$ of components and they are at least $2 n$.

Suppose that $K \triangle K^{\prime}=W(R)$. Since all components in $W(R)$ have the same area, Corollary 5.2 yields

$$
\frac{|W(R)|}{\left|K \cap K^{\prime}\right|} \leq h \cdot \max _{P \in \mathcal{P}_{h}} G(P) .
$$

From (54) we obtain the explicit bound $2 \sin ^{2}(\pi / h) / \cos (2 \pi / h)$, a decreasing function of $h$. Since $h \geq 2 n$ we get the following result.

Theorem 6.1. If $K$ and $K^{\prime}$ are two planar convex bodies with the same $X$-rays in $n$ different directions $(n \geq 3)$, and such that

i) $K \triangle K^{\prime}$ consists of a finite number of connected components of equal area, then

$$
\left|K \triangle K^{\prime}\right| \leq\left|K \cap K^{\prime}\right| \frac{1-\cos (\pi / n)}{\cos (\pi / n)}
$$


Equality holds if and only if, up to an affine transformation, the directions are equally spaced, $K$ and $K^{\prime}$ are congruent regular $n$-gons, and $K^{\prime}$ is $K$ rotated by $\pi / n$ about its center.

We remark that (74) is stronger than (73), via the classical isoperimetric inequality for $n$-gons.

The stability estimate can also be improved using more information about the set of directions, in case the error $\delta$ on the directions $\theta_{i}$ is small enough. This result and the extension to the general case, without assumption $i$ ) will be presented in a forthcoming paper by Dulio, Longinetti and Peri.

\section{REFERENCES}

[1] A. Barlotti, Una proprietà degli $n$-agoni che si ottengono trasformando in una affinità un $n$-agono regolare, Boll. Un. Mat. Ital. (3) $\mathbf{5 1 0}$ (1955), 96-98.

[2] G. Bianchi and M. Longinetti, Reconstructing Plane Sets from Projections, Discrete Comput. Geom. 5 (1990), 223-242.

[3] H. S. M. Coxeter, Affinely regular polygons, Abh. Math. Sem. Univ. Hamburg 62 (1992), 249-253.

[4] J. C. Fisher and R. E. Jamison, Properties of affinely regular polygons, in Geom. Dedicata 69 (1998), $241-259$.

[5] R. J. Gardner and P. McMullen, On Hammer's X-ray Problem, J. London Math. Soc. 21 (1980), 171-175.

[6] R. J. Gardner, Geometric Tomography, Cambridge University Press, Cambridge, 1995.

[7] O. Giering, Bestimmung von Eibeireichen und Eikörpen durch Steiner-Symmetrisierungen, Sber.Bayer.Akad. Wiss. München, Math.-Nat. Kl. (1962), 225-253.

[8] P. M. Gruber, Aspects of approximation of convex bodies, in Handbook of Convex Geometry (eds. P. M. Gruber and J. M. Wills), North-Holland, Amsterdam, 1993, 319-345.

[9] P. C. Hammer, 'Problem 2', Proc. Symp. in Pure Mathematics, Convexity, American Mathematical Society, VII (1963).

[10] G. Harel and J. M. Rabin, Polygons whose vertex triangles have equal area, Amer. Math. Monthly (2003), 606-619.

[11] F. John, Extremum Problems with Inequalities as Subsidary Conditions, Courant Anniversary Volume, Interscience, N.Y., 1948, 187-204.

[12] A. Kuba and G. T. Herman, Discrete Tomography: a historical overview, in Discrete Tomography (eds. G. T. Herman and A. Kuba), Birkhauser, Boston, MA, 1999, 3-34.

[13] M. Longinetti, An Isoperimeric inequality for convex polygons and convex sets with the same symmetrals, Geom. Dedicata 20 (1986), 27-41.

[14] M. Longinetti, Una proprietà di massimo dei poligoni affinemente regolari, Rendiconti del Circolo Matematico di Palermo 24 (1985), 448-459.

[15] M. Longinetti, Some questions of stability in the reconstruction of plane convex bodies from projections, Inverse Problems, 1 (1985), 87-97.

[16] M. A. Lopez and S. Reisner, Efficient approximation of convex polygons, Int. J. Comput. Geometry Appl., 10 (2000), 445-452.

[17] M. Ludwig, A characterization of affine length and asymptotic approximation of convex discs, Abh. Math. Semin. Univ. Hamb. 169 (1999), 75-88.

[18] A. Renyi and R. Sulanke, Über die konvexe Hülle von $n$ zufällig gewählten Punkten, Zeitschrift für Wahrscheinlichkeitstheorie 2 (1963), 75-84.

[19] A. Volčič, Well-posedness of the Gardner-McMullen reconstruction problem, Proc. Conf. Measure Theory, Oberwolfach 1983, Lecture Notes in Mathematics 1089, Springer, Berlin, 1984, 199-210. 
Istituto per le Applicazioni del Calcolo, Sezione di Firenze, Via Madonna del Piano - CNR Edificio F, 50019 - Sesto Fiorentino (FI), Italy

E-mail address: paolo@fi.iac.cnr.it

Dipartimento di Ingegneria Agraria e Forestale, Università degli Studi di Firenze, P.le DELle CASCINE 15 - 50139 Firenze - ItALY

E-mail address: marco.longinetti@unifi.it 